\section{Jahre Arbeitsgemeinschaft Minimal invasive Mammainterventionen (AG MiMi) in der DGS}

\author{
S. Paepke, P. Scheler, U. Peisker, I. Gruber
}

30 Jahre Deutsche Gesellschaft für Senologie und knapp 10 Jahre Arbeitsgemeinschaft Minimal invasive Mammainterventionen unter ihrem Dach; Gelegenheit für Dank und einen kurzen Rückblick.

Geboren wurde die Idee zur Gründung der AG MiMi in der Wüste, unter der Sonne Arizonas, dort tätig im Einüben neuer Fertigkeiten an neuer Technik; beeindruckt von der Stringenz, Genauigkeit und dem hohen wissenschaftlichen Anspruch unserer amerikanischen Kollegen, entwickelt aus dem praktisch-klinischen Tätigsein der Ultraschalldiagnostiker, Gynäkologen und Radiologen, orientiert an den damalig aktuellen Fragestellungen und dem Bedürfnis, diese konzentriert und vor allem interdisziplinär gemeinsam anzugehen.

Aus der daraus entstandenen Kommunikation entstand die Idee, die eher lose $\mathrm{Zu}$ sammenarbeit in einer Arbeitsgruppe zusammenzufassen. Was lag näher, als die Deutsche Gesellschaft für Senologie am Schreibtisch Prof. Wallwieners in Tübingen um Schirmherrschaft zu bitten.

Aus dem Erfahrungsschatz mehrerer Zentren, begleitet von einem wissenschaftlichen Beirat, wurden die Strukturen für die ersten Projekte geschaffen. Seitdem prägen die in den Jahresversammlungen vorgestellten Ideen die interdisziplinäre Projektarbeit:

Zeitlich parallel zur Etablierung des Deutschen Mammografiescrennings, das mit steigenden Anforderungen an die Mammadiagnostik und einer zunehmenden Verfügbarkeit stereotaktisch und sonografisch geführter Vakuumbiopsiesysteme einherging, wurde ein Indikationskatalog interdisziplinär abgestimmt und veröffentlicht [7].

Damit eng verknüpft waren die Projekte, die diagnostisch-therapeutische Entfernung benigner Befunde (Modell Fibroadenom) durch die sonografisch geführte Vakuumbiopsie [7] zu untersuchen und ebenso wie die Frage zu beantworten, ob Biopsien bei BIRADS-IV-Läsionen im Vergleich zu offen-chirurgischen Probeentnahmen ausreichend repräsentativ sind [3].

Der nächste Schritt, eine spannungsreiche und detaillierte Arbeit von 2 Jahren, war die Strukturierung, der Probelauf und die Einführung eines zentralen Biopsieregisters [5], ein wichtiges Organ für die Qualitätssicherung der minimalinvasiven Mammadiagnostik.

In den letzten Jahren wurde der zunehmende Trend zur Minimalisierung und Zielgerichtetheit diagnostischer Interventionen vorbereitet und wesentlich mitbestimmt durch Projekte der AG MiMi. Einen herausragenden Platz nimmt hier die Duktuskopie-Multizenterstudie [8] ein. Der technischen Verfügbarkeit wurden hier von Anfang an die umfassende Erhebung multizentrisch gesammelter Daten in hoher, aussagefähiger Fallzahl und die wissenschaftliche Analyse zur Seite gestellt. Parallel zu den genannten Projekten wurde ein weiterer Fokus auf die Fort- und Weiterbildung gelegt; Mammasonografie- und -interventionskurse und in bereits langer Tradition Duktuskopiekurse durchgeführt.

Die langjährige Projektarbeit der Arbeitsgemeinschaft Minimal invasive Mammainterventionen hat in enger Kooperation mit anderen Fachgremien der Deutschen Gesellschaft für Senologie die Entwicklung der Mammadiagnostik begleitet, wesentliche Entwicklungen früh erkannt, Daten gesammelt und ausgewertet und damit Leitlinienaussagen möglich gemacht, die mittlerweile Allgemeingut geworden sind.

Auf dem Fundament dieser Erfahrungen arbeiten wir an der nächsten Folge von Projekten, der Kryoablation benigner Befunde, Radiofrequenzablation von Mammatumoren, feinnadelaspirationszytologischer versus histologischer Dignitätssicherung des Sentinel-Lymph-Nodes und weiteren...

Wir freuen uns auf die Jubiläumstagung in Dresden und danken herzlich allen bisherigen Mitstreitern, Mentoren und Wegbegleitern!

\section{Literatur}

1 Grunwald S, Ohlinger R, Euler U et al. Minimalinvasive Diagnostik sezernierender Brusterkrankungen durch Milchgangsendoskopie. Endoskopie heute 2006; 18: 186189
2 Grunwald S, Heyer H, Paepke S et al. Endoskopische Milchgangsspiegelung - Indikationen und praktische Durchführung. Senologie 2006; 3: 122-127

3 Hahn M, Gall C, Baur A et al. Source of error in minimal-invasive interventions of the breast and how one can avoid it! Geburtsh Frauenheilk 2007; 67: 779-781

4 Hahn M, Krainick $U$, Peisker $U$ et al. Eignet sich das Hand Held Mammotome zur kompletten Entfernung benigner Läsionen der Brust? Arbeitsgemeinschaft für minimalinvasive Mammainterventionen (AG MiMi). Geburtsh Frauenheilk 2004; 64: 719-722

5 Hahn M, Gall C, Gruber I et al. Das zentrale Biopsieregister (ZBR) der Arbeitsgemeinschaft minimalinvasive Mammainterventionen (AG MiMi) der Deutschen Gesellschaft für Senologie (DGS) - Entwicklung einer Datenbankapplikation. Senologie 2008; 5: 264-268

6 Jacobs VR, Kiechle M, Plattner B et al. Breast ductoscopy with a $0.55-\mathrm{mm}$ mini-endoscope for direct visualization of intraductal lesions. J Min Invas Gyn L 2005; 12: 359-364

7 Kraninick-Strobel U, Huber B, Majer I et al. Complete extirpation of benign breast lesions with an ultrasound-guided vacuum biopsy system. Ultrasound Obst Gyn 2007; 29: 342-346

8 Ohlinger R, Frese H, Paepke S et al. Ultrasonographic compared to histologic sizing of benign and malignant breast lesions. Geburtsh Frauenheilk 2006; 66: 373-376

9 Paepke S, Ohlinger R, Kiechle M et al. Duktoskopie, Duktallavage, intraduktale Zytologie und Mikrobiopsie in der Diagnostik bei suspekter Mamillensekretion. Gyn Prakt Gyn 2007; 12: 415-422

\section{Bibliografie}

Dol http://dx.doi.org/

Senologie 2011; 8: 81

(c) Georg Thieme Verlag KG Stuttgart · New York . ISSN 1611-6453

\section{Korrespondenzadressen}

\section{Dr. med. Dipl. med. Stefan Paepke}

Frauenklinik und Poliklinik

der Technischen Universität München

Ismaningerstr. 22

81675 München

stefan.paepke@|rz.tu-muenchen.de

\section{Dr. med. Peter Scheler}

Chefarzt des Brustzentrums

St.-Vincenz-Krankenhaus

Auf dem Schafsberg

65549 Limburg

p.scheler@st-vincenz.de

\section{Dr. med. Uwe Peisker}

Chefarzt der Frauenklinik

Hermann-Josef-Krankenhaus

Tenholterstr. 43

41812 Erkelenz

upeisker@hjk-erkelenz.de

\section{Dr. med. Ines V. Gruber}

Universitätsfrauenklinik Tübingen

Calwerstr. 7

72076 Tübingen

agmimi@senologie.org 